\title{
Developmental phenotyping goes on auto with LAMA
}

Horner, N.R. et al. Development (2021). https://doi.org/10.1242/dev.192955

Since 2011, members of the International Mouse Phenotyping Consortium (IMPC) have been making their way through the mouse genome, knocking out genes one-by-one and cataloging the resulting phenotype. To date, they've made it through over 7000 genes, about a third of which have markedly deleterious roles on developing mice.

Phenotyping mutants across embryonic development can yield temporal insight into developmental processes and how they go awry, but manual annotation can take hours. With thousands of whole-embryo micro-CT scans of mice from mutant lines taken at multiple developmental time points waiting to be phenotyped, the IMPC wanted to pick up the pace.

Writing in the journal Development, Neil Horner, a post-doc at the Medical Research Council Harwell Institute, and colleagues collaborating with the IMPC describe an automated pipeline for phenotyping mouse embryos: the Lightweight Analysis of Morphological Abnormalities, or LAMA.
"LAMA was designed to be quicker, and one way we address this is to distribute tasks across multiple machines to speed up analysis," Horner says. "Also, the approach we use in registering our images allows us to make use of large amounts of baseline data to increase statistical power, which helps greatly as many IMPC mutant lines have low sample number."

LAMA compares individual scans against a population average of wild-type embryos at a particular developmental stage. In the paper, the team used scans of 8 male and 8 female wild-type mice at E14.5 to create a novel atlas with 103 labels, which they then used to phenotype two IMPC mutant lines. For $W f d c 2^{-/-}$mice, which have severe pulmonary phenotypes at perinatal day 1.5 , LAMA noted that the knockouts have smaller tracheas and bronchi at E14.5 than wild-type embryos. LAMA also captured previously reported bone - but not lung - abnormalities for $A C A N^{-/}$knockouts at E14.5. Looking at wild-type embryos, Horner was surprised to see significant sex effects in the eye - male lenses were slightly larger than those of females, a detail that can be missed when just eyeballing the scans. "I'm not sure of the biological significance of this finding, but that's what the results show us," he says.

The IMPC is currently adding developmental stages while working to optimize the registration process. "Getting registration parameters that work for each developmental time point can be tricky. A set of parameters that work well for some organs can lead to inaccuracies in others. So it can be a matter of finding a set that represent a good compromise of registration accuracy across the whole embryo," says Horner. Manual checks are advised, and he also suggests interested users take advantage of LAMA's QC tools to keep their own phenotyping process speeding along. LAMA can be found online on github.

\section{Ellen P. Neff}

Published online: 22 March 2021

https://doi.org/10.1038/s41684-021-00746-7

\section{natureresearch} ACADEMIES

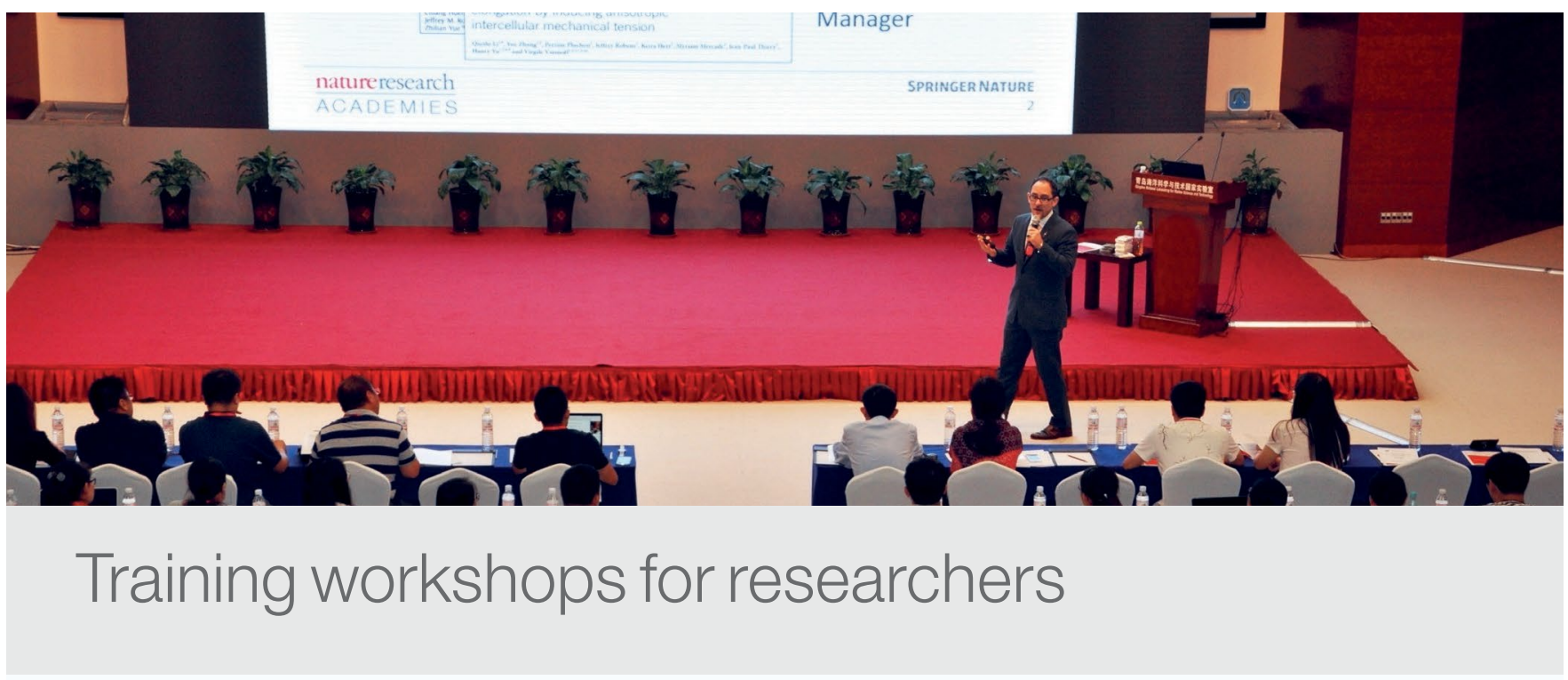

A series of workshops to support researchers, covering topics such as getting published, journal editing, clinical research methodology, and applying for research positions.

Visit partnerships.nature.com/academies to host an academy at your institution. 